\section{References}

1 Edwards LB, Acquaviva FA, Livesay VT et al. An atlas of sensitivity to tuberculin, PPD-B, and histoplasmin in the United States. Am Rev Respir Dis 1969;99:1-132.

2 Mukherjee JJ, Villa ML, Tan L et al. Bilateral adrenal masses due to histoplasmosis. J Clin Endocrinol Metab 2005;90:6725-6.

3 Goodwin RA, Jr., Shapiro JL, Thurman GH et al. Disseminated histoplasmosis: clinical and pathologic correlations. Medicine (Baltimore) 1980;59:1-33.
4 Sarosi GA, Voth DW, Dahl BA et al. Disseminated histoplasmosis: results of long-term follow-up. A center for disease control cooperative mycoses study. Ann Intern Med 1971;75:511-6.

Address for correspondence: Dr U Srirangalingam, Department of Endocrinology, St Bartholomew's Hospital, West Smithfield, London EC1M 7BE.

Email: u.srirangalingam@qmul.ac.uk

\title{
Acute renal failure in diabetes: looking beyond diabetic retinopathy
}

\author{
Piya Sen Gupta, Megan Crofts, Kate Antrobus and John V Anderson
}

\section{Case presentation}

A 43-year-old construction worker was admitted to the emergency department with increasingly severe right-sided abdominal pain of three days' duration. There had been no precipitant injury and it had not improved with regular ibuprofen. He had type 1 diabetes of 20 years' duration treated with once daily basal glargine and mealtime NovoRapid ${ }^{\circledR}$ insulin. Other medications were simvastatin $80 \mathrm{mg}$ od, losartan $25 \mathrm{mg}$ od and perindopril $4 \mathrm{mg}$ od. His complications of diabetes included bilateral photocoagulation-treated retinopathy but no known peripheral neuropathy or nephropathy. He also had hypertension, smoked 10 cigarettes daily and had a pleurodesis procedure following a pleural drain for a parapneumonic effusion five years previously.

Examination revealed an alert and lucid (Glasgow Coma Score (GCS) 15/15) Causasian man with evidence of hypovolaemia (dry mucous membranes), cool peripheries, temperature $36.0^{\circ} \mathrm{C}$, pulse $86 / \mathrm{min}$, blood pressure $139 / 87 \mathrm{mmHg}$ and respiratory rate $15 / \mathrm{min}$. Cardiac examination was normal but chest examination revealed dullness to percussion and reduced breath sounds at the left base. He had a distended abdomen with right flank tenderness. Baseline admission investigations are shown in Box 1.

Piya Sen Gupta, diabetes registrar; Megan Crofts, core medical trainee 2; Kate Antrobus, foundation year 2; John V Anderson, consultant diabetologist

Department of Diabetes, Homerton University Hospital, London

\section{What is the differential diagnosis and the most likely diagnosis?}

His main problems are abdominal pain and renal failure on a background of chronic type 1 diabetes. With no known previous nephropathy, it is safest to assume this is acute kidney injury (AKI), the causes of which can be divided into:

- pre-renal or volume responsive

- intrinsic renal

- post-renal subcategories. $^{1}$

The likelihood of each differential within these divisions is influenced by the specifics of this case.

With his type 1 diabetes, electrolyte abnormalities and abdominal pain as a main presenting feature, diabetic ketoacidosis

\section{Key learning points}

- Acute kidney injury (AKI) requires prompt and careful identification of the underlying cause.

- The principles of AKI management begin with the cessation of further renal insult; careful assessment of volume status is also important.

- Rapidly progressive intrinsic renal disease is rare but it is important to diagnose so that directed therapy can follow.

- Although patients with longstanding diabetes can have chronic kidney disease, 'diabetic nephropathy' as the cause of renal failure should never be assumed without confirmation.

- Renal function decline in diabetes warrants urgent establishment of whether this is a new phenomenon by direct patient questioning and checking hospital and primary care records. 
Box 1. Test results.

\section{Baseline}

\begin{tabular}{|c|c|c|}
\hline \\
\hline & & Reference ranges \\
\hline Sodium & 129 & (135-147 mmol/l) \\
\hline Potassium & 6.5 & $(3.4-4.9 \mathrm{mmol} / \mathrm{l})$ \\
\hline Urea & 18.7 & $(2.0-6.6 \mathrm{mmol} / \mathrm{l})$ \\
\hline Creatinine & $309^{*}$ & (75-125 $\mu \mathrm{mol} / \mathrm{l})$ \\
\hline${ }^{*}$ c.f. six months ago & 95 & \\
\hline Estimated glomeruler filtration rate & 19 & \\
\hline Glucose & 14.9 & $(4-7 \mathrm{mmol} / \mathrm{l})$ \\
\hline Haemoglobin & 8.2 & $(13.0-18.0 \mathrm{~g} / \mathrm{dl})$ \\
\hline Mean cell volume & 80.9 & $(80-98 \mathrm{fl})$ \\
\hline White cell count & 10.1 & $\left(4.0-11.0 \times 10^{9} / 1\right)$ \\
\hline Platelets & 443 & $\left(150-600 \times 10^{9} / I\right)$ \\
\hline International normalised ratio & 1.1 & $(1.1-1.5)$ \\
\hline \multirow[t]{5}{*}{ Urinalysis } & Protein & $3+$ \\
\hline & Blood & $2+$ \\
\hline & Glucose & + \\
\hline & Ketones & + \\
\hline & Leucocytes & Negative \\
\hline Arterial gas & pH 7.42, pO2 10.7, pCO2 3.8 & \\
\hline$\left(\mathrm{FiO}_{2} 21 \%\right)$ & HCO3 20.7, BE-6.3 & \\
\hline Electrocardiogram & Sinus rhythm. Rate 70 bpm. & \\
\hline Chest radiograph & $\begin{array}{l}\text { Left sided pleural effusion. Right basal patchy } \\
\text { shadowing tracking into the fissure. }\end{array}$ & \\
\hline \multirow[t]{2}{*}{ Ultrasound of the kidneys } & $\begin{array}{l}\text { Hypereflective kidneys with loss of } \\
\text { corticomedullary differentiation. }\end{array}$ & \\
\hline & Further results & \\
\hline Erythrocyte sedimentation rate & 81 & $(<5 \mathrm{~mm} / \mathrm{hr})$ \\
\hline $\mathrm{HbA}_{1 \mathrm{c}}$ & 10.4 & $(<6.5 \%)$ \\
\hline Creatine kinase & 78 & $(50-220 \mathrm{U} / \mathrm{I})$ \\
\hline Triglyceride & 2.0 & $(<1.7 \mathrm{mmol} / \mathrm{l})$ \\
\hline Amylase & 20 & $(<120 \mathrm{U} / \mathrm{I})$ \\
\hline Bilirubin & 4 & (3-17 $\mu \mathrm{mol} / \mathrm{l})$ \\
\hline Aspartate transaminase & 14 & (5-40 IU/LI \\
\hline Alkaline phosphatase & 95 & (40-130 IU/I) \\
\hline Calcium & 2.45 & $(2.2-2.6 \mathrm{mmol} / \mathrm{l})$ \\
\hline Phosphate & 1.36 & $(0.8-1.5 \mathrm{mmol} / \mathrm{l})$ \\
\hline Albumin & 19 & $(35-50 \mathrm{~g} / \mathrm{l})$ \\
\hline Total protein & 51 & $(60-80 \mathrm{~g} / \mathrm{l})$ \\
\hline 24-hr urinary protein & 1.41 & $(<0.15 \mathrm{~g} / \mathrm{l})$ \\
\hline Complement C3 & 1.45 & $(0.75-1.65 \mathrm{~g} / \mathrm{l})$ \\
\hline C4 & 0.29 & $(0.14-0.54 \mathrm{~g} / \mathrm{l})$ \\
\hline ANA, ENA, GBM & negative & \\
\hline ANCA & positive pANCA, MPO57 1:20 titre & \\
\hline Echocardiogram & No valvular vegetations, & \\
\hline
\end{tabular}

$\mathrm{ANA}=$ antinuclear antibody; ANCA = antineutrophil cytoplasmic antibody; ENA = extractable nuclear antigen; GBM = glomerular basement membrane. 

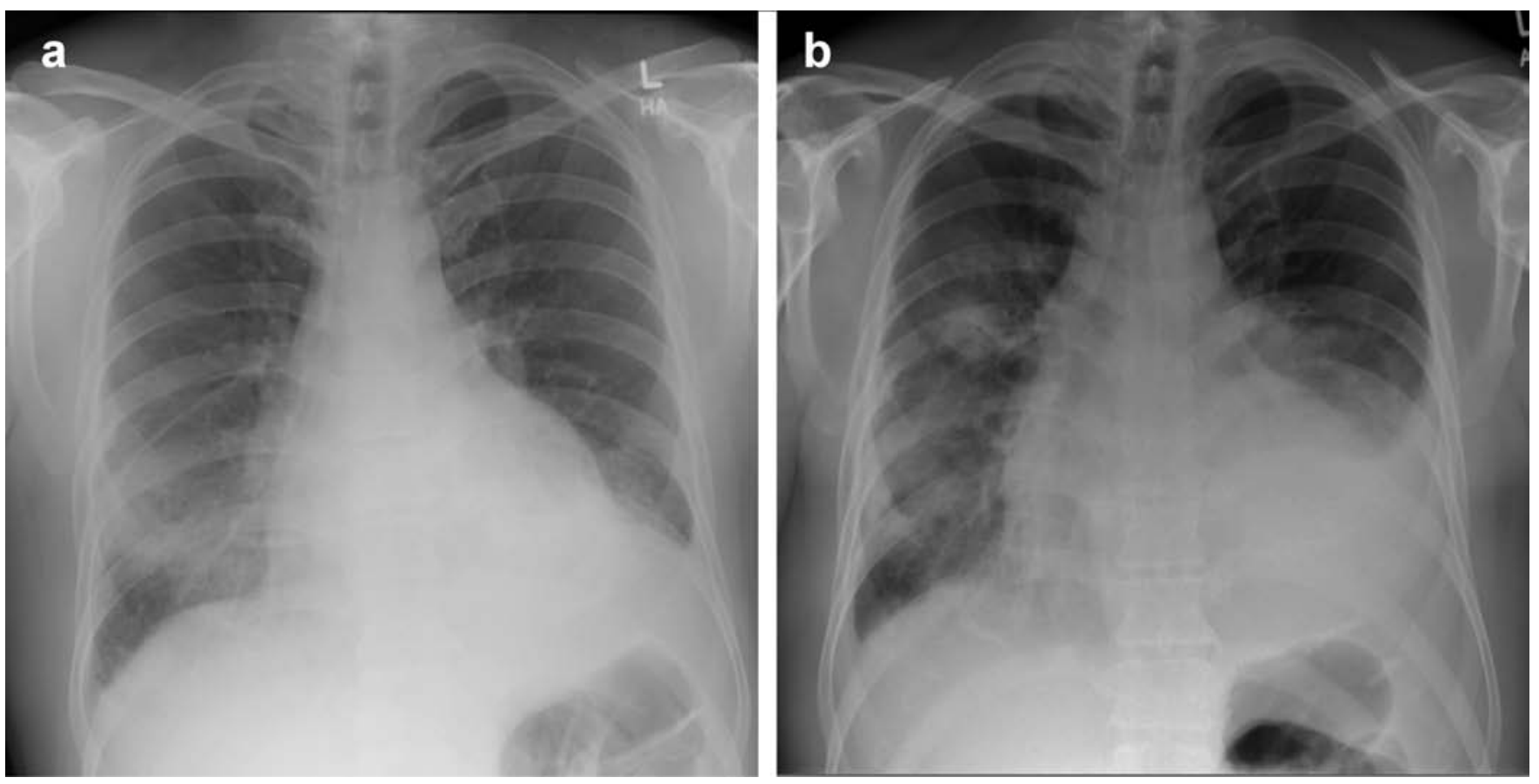

Fig 1. Chest radiographs. (a) at admission, (b) seven days after shows worsening right consolidation, bilateral pleural effusions and pericardial effusion.

should be, and can be, quickly ruled out. Adrenal insufficiency and pancreatitis secondary to hypertriglyceridaemia should also be considered.

Pre-renal causes account for the majority (75\%) of AKI cases. ${ }^{2}$ Although pre-renal AKI may be partially or wholly responsible here, eg 'surgical' abdomen or sepsis from a right basal pneumonia, his clinical volume status examination did not suggest severe hypovolaemia and he was not overtly peritonitic.

There was little to suggest a post-renal cause, which causes AKI infrequently and usually on a background of a known obstructive urological process. He maintained a good urine output and the character of his abdominal pain was not typical for renal calculi or urinary obstruction.

The most likely differential diagnosis is intrinsic renal disease causing AKI. This could be due to:

- glomerular damage from glomerulonephritis in keeping with the heavy proteinuria

- glomerular endothelial damage from vasculitis which could affect multiple organs given his previous and current chest pathology

- tubular damage from acute tubular necrosis or rhabdomyolysis (see below)

- intersitial nephritis secondary to recent non-steroidal antiinflammatory drug use.

Other intrinsic renal causes that should be borne in mind are diabetic nephropathy and rhabdomyolysis. Proteinuria and microscopic haematuria with his history of retinopathy and poor glycated haemoglobin $\left(\mathrm{HbA}_{1 \mathrm{c}}\right)$ result make diabetic nephropathy a possibility, but the rapid deterioration in renal function counts against this. Rhabdomyolysis secondary to his diabetes or statin use (particularly given the high dose) may cause pain and haematuria representing myoglobinuria, so his creatine kinase should be checked.

\section{What is the initial management?}

The principles of his initial management begin with limiting any further insult to his kidneys by discontinuing all potentially offending drugs (ibuprofen, simvastatin, losartan) and selecting non-nephrotoxic analgesia. Careful assessment of his volume status and meticulous regular attention to his fluid balance including urine output monitoring is needed, with any fluid deficits rectified.

The underlying cause should be sought in order to commence directed treatment. An ultrasound or computed tomography (non-contrast) scan of his renal tract will identify most postrenal AKI causes and 'renal screen' bloods should also be arranged as a matter of urgency including antinuclear antibody, antineutrophil cytoplasmic antibody (ANCA), extractable nuclear antigen, antiglomerular basement membrane antibodies, immunoglobulins, erythrocyte sedimentation rate, creatine kinase, hepatitis B and C, and HIV. Thromboprophylaxis should also be commenced.

Considerations with respect to his diabetes include ensuring insulin treatment continuation, examining his feet to eliminate possible septic foci and performing fundoscopy 
to assess for further proliferative retinopathy. It is important to establish his usual diabetes control levels and previous renal function.

\section{Case progression}

Following admission, the hyponatraemia and hyperkalaemia improved. His renal function initially improved with intravenous hydration (creatinine $250 \mu \mathrm{mol} / \mathrm{l}$ ) suggesting a pre-renal element but subsequently deteriorated rapidly. Widespread peripheral oedema developed but his urine output remained good. He developed fevers, worsening right-sided lung consolidation (Fig 1) and was treated with antibiotics appropriate for pneumonia. Further results are shown in Box 1. He was transferred to the renal team for a renal biopsy and further management. His diagnosis was ANCA positive vasculitis. He was treated with high-dose steroids and cyclophosphamide and discharged to his local diabetes-renal unit for follow-up. On discharge, his creatinine was stable at $420 \mu \mathrm{mol} / \mathrm{l}$ (chronic kidney disease stage $\mathrm{V}$ ).

\section{Discussion}

ANCA positive vasculitis (APV) encompasses a group of smallvessel vasculitides: Wegener's granulomatosis (WG), Churg Strauss syndrome (CSS) and microscopic polyangiitis (MPA), which are commonly associated with ANCA. The annual incidence of APV in the UK is 10-20 per million, rising with age to peak in those aged $65-74$ years. ${ }^{3}$ Clinical features vary depending upon the organs affected, but all include general constitutional symptoms of fever, fatigue, anorexia, weight loss and night sweats. WG has a predilection for the respiratory tracts, giving rise to sinusitis, rhinorrhoea and epistaxis with upper respiratory tract involvement and pulmonary haemorrhage with lower tract involvement. ${ }^{4}$ Renal involvement is common in all three of the ANCA positive disease entities being more severe in WG and MPA, as in this case. Although less common presentations of APV can involve ocular, cardiac, peripheral nervous and gastrointestinal systems, there was no evidence of gastrointestinal haemorrhage to account for his abdominal pain, which is an unusual presenting feature. The diagnosis of APV is based on clinical findings, biopsy results and the presence of ANCA. In this case, the patient's longstanding diabetes complicated the clinical findings.
Prognosis of APV is poor with a $90 \%$ two-year mortality if left untreated. It requires prompt diagnosis and immune modulation to avoid long-term renal scarring. Prednisolone and cyclosphosphamide constitute standard therapy and control APV in $80-90 \%$ of patients. However, one third of patients relapse within two years. The treatment regimes are associated with significant morbidity including bladder and lymphoproliferative malignancies, infertility and diabetes. ${ }^{4}$ Although development of diabetes was less of a concern with this patient, his poorly controlled longstanding type 1 diabetes with proliferative retinopathy complications and history of smoking will also impact on his long-term prognosis.

AKI itself is associated with increased risk of mortality and has thus led to the development of the RIFLE (defining the at risk, injury, failure, loss or end-stage kidney) classification ${ }^{5}$ and AKIN (stage 1-3) staging guidelines. ${ }^{1}$ These offer a means of categorising the extent of kidney damage based on the serum creatinine, glomerular filtration rate and urine output with the aim of identifying renal insufficiency earlier to limit potential permanent injury.

In summary, this case presents a man with longstanding type 1 diabetes who developed AKI. Although his renal function was initially volume responsive suggesting a pre-renal element, the subsequent rapid deterioration in renal function warranted urgent diagnosis and directed treatment with immune-modulation for ANCA positive vasculitis.

\section{References}

1 The Renal Association. Acute kidney injury clinical practice guidelines. London: The Renal Association, 2008.

2 Nash K, Hafeez A, Hou S. Hospital-acquired renal insufficiency. Am J Kidney Dis 2002;39:930-6.

3 Watts RA, Scott DGI. Epidemiology of systemic vasculitis. In: Bridges L, Ball G (eds), Vasculitis, 2nd edn. Oxford: Oxford University Press, 2007:7-21.

4 Kamesh L, Harper L, Savage COS. ANCA-positive vasculitis. J Am Soc Nephrol 2002;13:1953-60.

5 Bellomo R, Ronco C, Kellum JA, Mehta RL, Palevsky P; Acute Dialysis Quality Initiative workgroup. Acute renal failure: the Second International Consensus Conference of the Acute Dialysis Quality Initiative (ADQI) Group. Crit Care 2004;8:R204-12.

Address for correspondence: Dr P Sen Gupta,

Department of Diabetes, Homerton University Hospital, Homerton Row, London E9 6SR.

Email: psengupta79@doctors.net.uk 